\title{
Uso del video en el aprendizaje del taekwondo: estudio en niños en edad escolar
}

\author{
César A. Bernal-Torres ${ }^{(1) \star}$, John D. Hoyos ${ }^{(2)}$, Germán Fracica ${ }^{(3)}$ y Fiorela A. Fernández-Otoya(4) \\ (1) Escuela Internacional de Ciencias Económicas y Administrativas, Universidad de La Sabana, Km. 7, Autopista Norte \\ de Bogotá, Puente del Común, Campus Universitario, Chía, Colombia. (correo-e: cesar.bernal@unisabana.edu.co). \\ (2) Unidad de Educación, Vicerrectoría Regional, Corporación universitaria minuto de Dios, Bogotá Sur Calle 1 N 9-50 Sur \\ Bogotá, Colombia (correo-e: jhoyoscifue@uniminuto.edu.co) \\ (3) Programa de Maestría en Educación, Universidad de La Sabana, Km. 7, Autopista Norte de Bogotá, Puente del Común, \\ Campus Universitario, Chía, Colombia. (correo-e: german.fracica@gmail.com). \\ (4) Universidad Católica Santo Toribio de Mogrovejo, Escuela de Educación, Av. San Josemaría Escrivá 855, Grupo de \\ Investigación en Informática Educativa y TIC, Chiclayo 14012, Perú. (correo-e: ffernandez@usat.edu.pe)
}

${ }^{*}$ Autor a quien debe ser dirigida la correspondencia.

Recibido Jun. 19, 2019; Aceptado Ago. 19, 2019; Versión final Sep. 26, 2019, Publicado Feb. 2020

\section{Resumen}

Este artículo analiza los beneficios del uso del video en el proceso aprendizaje del taekwondo en niños en edad escolar y la relación entre la motivación por el uso de esta tecnología y otros beneficios pedagógicos de ésta. Para ello se realizó un experimento con un grupo de 42 niños de entre 7 y 12 años cuyos resultados indican que el uso de los videos es un factor generador de alta motivación en los niños para realizar la práctica de ese deporte. Sin embargo, esa motivación solo se relaciona con la mejora en la técnica en la ejecución de las figuras y la corrección de los errores de ejecución en el combate y no con los demás beneficios del uso del video. El estudio pretende contribuir a la comprensión de las implicaciones del uso del video en el proceso de aprendizaje y el rol de la motivación en ese proceso.

Palabras clave: niños; proceso de aprendizaje; estrategia pedagógica; taekwondo, video

\section{Use of video in taekwondo learning: a study in children in school age}

\begin{abstract}
This article analyzes the benefits of the use of video in the learning process of taekwondo in children in school age and the relationship between motivation by the use of this technology and other pedagogical benefits of this. For this, an experiment with a group of 42 children aged 7 to 12 was performed. The results indicate that the use of videos is a generator factor of high motivation in children to make the practice of this sport, However, this motivation is relates only with the improvement of the technique in the execution of the figures and the correction of the errors of execution in combat and not with the other benefits of the use of video. The study aims to contribute to the understanding of the implications of the use of video in the learning process and the role of motivation in that process.
\end{abstract}

Keywords: children; learning process; pedagogical strategy; taekwondo; video 


\section{INTRODUCCIÓN}

Es indudable la importancia que cada día adquiere el uso de las tecnologías de la información y las comunicaciones (TIC) en el proceso de enseñanza dado que éstas facilitan a los estudiantes la adquisición de nuevas competencias y permiten mejorar la labor didáctica de los docentes. En ese sentido, el video es considerado como una estrategia pedagógica que mejora el proceso de aprendizaje (García, 2017); su uso, aumenta la posibilidad de ampliar el trabajo innovador en dicho proceso. En el caso específico del aprendizaje del taekwondo, como deporte que contribuye al desarrollo integral de los niños, las tecnologías y, en especial, el video facilita la asimilación del aprendizaje del estudiante, genera un ambiente motivacional y creativo debido a su poder atractivo y contribuye a mejorar las técnicas de ejecución del deporte en mención.

Por lo antes descrito, y dado que en Colombia y, en general, en Latino América son pocos los estudios que se han realizado sobre los impactos del uso del video en la práctica de los deportes en los niños, esta investigación se orientó a analizar sus beneficios en el proceso de aprendizaje de las prácticas del taekwondo en un grupo de niños y, también, a analizar la relación entre la motivación generada por el uso de esta tecnología y los demás beneficios de la misma dado que en diversos estudios sobre motivación y aprendizaje se suele mencionar la relación positiva entre la motivación generada por el uso de las tecnologías y sus diferentes beneficios en el mencionado proceso; sin embargo, los resultados de diversos estudios en el campo de la psicología y la pedagogía indican que dicha relación no es evidente (Schunk, y Zimmerman, 2012). Esto con el propósito de aportar evidencia empírica sobre el tema y contribuir así a la comprensión y el fortalecimiento del uso de las TIC en el proceso de aprendizaje, en particular en el campo del deporte, que es una excelente estrategia para promover el desarrollo integral de los niños y, en general, de cualquier persona.

Según Orozco (2018), el deporte pedagógicamente bien orientado contribuye al desarrollo integral de las potencialidades de sus practicantes. Así, el deporte como medio educativo brinda la posibilidad de orientar a los estudiantes en la formación de la personalidad, la motivación y, en general, en la formación integral, es decir, la buena práctica del deporte hace un gran aporte en la formación de valores de las personas desde los niños a los adultos (Carbou, 2016). Combinar la carrera deportiva con la formación académica es una gran estrategia pedagógica para mejorar el proceso aprendizaje en las instituciones académicas. La práctica de deportes de contacto ha sido incluida en las actividades deportivas de diversas instituciones educativas con excelentes beneficios para la formación de los estudiantes. El taekwondo y el kick boxing educativo son una muestra de esto (Menéndez, 2016). Como práctica pedagógica, el taekwondo se enmarca en los deportes con gran sentido formativo ya que sobresale en su característica filosófica a través de los principios de cortesía, integridad, perseverancia, autocontrol y espíritu indomable (da Silva Soares, 2016).

El taekwondo, como deporte de contacto directo, es incluido como práctica de la educación física en la formación en diversas instituciones académicas de diversos países, respetando los limites fisiológicos y psicológicos del alumno (Grigore, 2015 y Casolino et al., 2012) porque su práctica como medio formativo es una estrategia que garantiza los objetivos educativos por la variedad de posibilidades pedagógicas que ofrece dado que, como deporte, permite orientar a los estudiantes en valores, en la formación de la personalidad, en la motivación y en el manejo y control de la ansiedad por parte de los practicantes (González y González, 2017). De acuerdo con García (2014) y Bridge et al. (2014), la práctica del taekwondo resulta ser una estrategia clave que contribuye al proceso de formación humana integral de los estudiantes (niños y adolescentes) porque en esta práctica se consideran las dimensiones sociales, éticas, psicológicas, fisiológicas y cognitivas del practicante, dimensiones estas que requieren el compromiso de toda persona que pretenda lograr un desarrollo armónico e integral.

Según Mendoza y Placencia (2017), el uso, en los procesos educativos, de las TIC facilitan a los estudiantes la adquisición de nuevas competencias y a los docentes les permite mejorar en su labor académica. En general, según Guillen et al. (2018), en el campo educativo, las TIC contribuyen a crear experiencias que enriquecen el proceso de enseñanza-aprendizaje con beneficios para el conjunto de la comunidad académica. En este sentido, la introducción de las TIC en el ámbito educativo es considerado un factor clave para el progreso y el desarrollo de la sociedad, que contribuye a impulsar numerosas iniciativas de todo orden, tanto en el ámbito nacional como en el internacional (Del Moral et al., 2011y Huapaya et al., 2005). Al respecto, Zambrano et al. (2017) resaltan la importancia de contar con un buen diseño pedagógico para el uso de las TIC en el campo educativo.

Una de las estrategias pedagógicas soportadas en las TIC para la mejora de los procesos de la enseñanza y el aprendizaje es el video (García, 2017). En este mismo sentido, Tobías-Martínez (2015) enfatiza la importancia del uso del video como recurso didáctico para estimular la construcción de nuevo conocimiento en los procesos de enseñanza y aprendizaje. De igual forma, Monedero (2015) afirma que el uso del video aumenta la posibilidad de ampliar el trabajo innovador en los procesos de la enseñanza y el aprendizaje. 


\section{OTROS ANTECEDENTES}

Varios autores sostienen que la incorporación de la tecnología está marcada por la motivación que el sujeto o esa tecnología generen (Osuna, 2018; Wei y Weng, 2015; Lu y Ying-Chieh, 2014); y Di Serio, 2013). En esta dirección, Bernaus et al. (2009) y Pearson y Moarnaw (2005) sostienen que existe relación positiva entre la motivación generada por el alumnado frente a una estrategia pedagógica y los resultados de su aprendizaje. Por esto, se considera de vital importancia el uso de estrategias pedagógicas que motiven en el alumno el interés por su aprendizaje (Sanchez-Otero et al., 2019; Grissom et al., 2013). Sin embargo, esta relación entre el factor motivacional por el uso de las TIC y los demás resultados de los procesos de la enseñanza y el aprendizaje no siempre es clara dado que estos, son procesos complejos que involucran diversos aspectos tanto del que aprende y del que enseña como del contexto en el que se dan estos procesos (Martínez-Argüello et al., 2018; Schunk y Zimmerman, 2012; Jaclyn, 2010; Traveset, 2007).

Para García (2017) y Álvarez (2016), en el caso específico del aprendizaje del taekwondo, las tecnologías y, en especial, el entorno multimedia facilitan el aprendizaje constructivista porque el mismo estudiante contribuye con la mejora en el desarrollo de técnicas de ejecución puesto que las imágenes proyectadas mediante el uso del video despiertan su poder atractivo generando un ambiente motivacional y creativo y porque, además, el video puede ser usado como herramienta de aprendizaje o de evaluación, según el contexto que el maestro quiera direccionar. Sobre esa posibilidad de análisis del contenido, Méndez (2018) indica que el video es un recurso valioso para explicar paso a paso un contenido teórico y así facilitar su enseñanza y su aprendizaje. A este respecto, el uso del video como herramienta pedagógica para el aprendizaje es un gran aporte a los modelos tradicionales de la enseñanza del deporte del taekwondo. Así, el cambio de paradigmas en los procesos de enseñanza y aprendizaje ofrece una serie de herramientas en las que el estudiante no solo aprende para sí sino que tiene la posibilidad de compartir y contrastar lo aprendido con otros. Esa posibilidad de intercambio de ideas motiva fuertemente su aprendizaje.

De acuerdo con Guillén et al. (2017), en todo proceso de enseñanza y de aprendizaje de los deportes por parte de los niños, en particular de los deportes de contacto, como el taekwondo, es importante que el componente metodológico se apoye en el uso de tecnologías de multimedia que faciliten el aprendizaje de la técnica y la táctica. En este sentido, teniendo en cuenta la importancia de la práctica del taekwondo en la formación integral de los niños y los jóvenes y del uso del video como estrategia para la mejora de los procesos de enseñanza y el aprendizaje de este deporte, esta investigación tuvo como objetivo evidenciar los beneficios del uso del video en el proceso de enseñanza y aprendizaje de las prácticas del taekwondo en un grupo de niños aprovechando que estos son altamente receptivos al uso de las diferentes herramientas tecnológicas (Córdoba et al., 2017).

\section{METODOLOGÍA}

Esta investigación, de carácter preexperimental, utilizó el video como herramienta tecnológica y pedagógica por los múltiples beneficios didácticos que ofrece al proceso de enseñanza y de aprendizaje (García, 2017), En este sentido, se reconoce que, por su nivel preexperimental (exploratorio) los resultados aquí obtenidos tienen limitaciones de validez interna y externa (Martin y Martin, 2008) debido a que no se utilizó aleatoriedad para la participación de los niños en el proyecto, tampoco se utilizó pretest, ni se consideró la presencia de grupos de control para definir la eficacia de sus resultados, sin embargo, esos resultados son un buen indicativo de la evaluación sistemática de las variables analizadas, aunque, sin potencial de generalización a otras poblaciones. Para sustituir el pretest, se contó con información de parte de los instructores acerca del nivel de prácticas del Taekwondo que tenían los niños antes de iniciar el preexperimento y, para corregir en gran parte la ausencia de grupos de control, al final del experimento, igualmente se encuestó a los instructores que guiaron los ejercicios del uso del video para conocer su percepción sobre la diferencia de resultados entre el antes y el después de esos ejercicios.

\section{Sujetos}

La información se obtuvo de un total de 42 niños (todos hombres) de edades entre 7 y 12 años que en el momento de la investigación tenían entre 12 y 18 meses de asistencia a prácticas formales de taekwondo dirigidas por un maestro en ese deporte. Los niños participantes del estudio eran estudiantes formales de dos instituciones académicas, de carácter privado, localizadas en la ciudad de Bogotá y que ofrecen la práctica del taekwondo como parte de la formación deportiva de los niños. Los ejercicios con los niños se realizaron en las instalaciones de los respectivos colegios en horarios de sus clases formales de deporte (horas de la mañana).

Para lograr la participación de los niños se invitaron seis instituciones de formación académica tradicional (colegios) en la que se ofrece la práctica del taekwondo y después de varias reuniones con los directivos de 
esas instituciones y con sus instructores de taekwondo, a quienes se presentó el objetivo de la investigación y del uso que se le daría a la información, sólo dos de esas instituciones concretaron su participación. En total, el proyecto comenzó con la participación de 53 niños, distribuidos en cuatro grupos: grupo $1=15$ niños, grupo $2=12$ niños, grupo $3=11$ niños y grupo $4=15$ niños, los cuales una vez finalizado el proyecto y depurados los datos con el requisito de la asistencia de cada niño al $100 \%$ de las actividades programadas con los videos y responder de forma completa la encuesta, los grupos quedaron así: grupo $1=13$ niños, grupo $2=10$ niños, grupo 3 niños = 8 y grupo $4=11$ niños. Vale mencionar que, tanto a cada uno de los niños, como a los instructores y los directivos de las instituciones participantes se les aclaró que la participación en el proyecto era totalmente voluntaria, anónima y que los resultados de este tenían propósitos eminentemente académico.

\section{Instrumento - cuestionario}

Al finalizar los cuatro meses de las prácticas del deporte, con apoyo del video, a los niños se les aplicó una encuesta conformada por diez enunciados, en escala Likert de 1 a 5, en donde se averiguó sobre la percepción de los niños de los beneficios del uso del video para la práctica del taekwondo. El cuestionario se elaboró a partir de la fundamentación teórica expuesta en la revisión de literatura, especialmente, en los planteamientos de García (2017) y Álvarez (2016), quienes afirman que, en el caso específico del aprendizaje del taekwondo, las tecnologías y, en especial, el entorno multimedia facilitan su aprendizaje porque el mismo estudiante contribuye con la mejora en el desarrollo de las técnicas de ejecución puesto que las imágenes proyectadas mediante el uso del video despiertan su poder atractivo generando un ambiente motivacional y creativo y porque, además, el video puede ser usado como herramienta de aprendizaje o de evaluación, según el contexto que el maestro quiera direccionar. También en los de Méndez (2018) quien indica que, el video es un recurso valioso para explicar paso a paso la práctica de un deporte y así facilitar su enseñanza y su aprendizaje.

Previo a su aplicación, dicho cuestionario fue sometido a juicio de cuatro expertos, quienes en general, basados en el objetivo del proyecto y en los rasgos de los participantes (destinatarios) del mismo, realizaron observaciones que llevaron a ajustar el formato inicial de 15 ítems a solo 10 relacionados específicamente con la buena práctica de ese deporte. También se realizó prueba de fiabilidad mediante el Alpha de Cronbach el cual arrojó un valor de 0,803 , indicando con esto que el cuestionario final es confiable para su aplicación. De otra parte y, con el propósito de contrastar los resultados de la encuesta aplicada a los niños, de forma simultánea, se aplicó un cuestionario de preguntas abiertas a cada uno de los instructores de taekwondo que dirigieron el ejercicio del uso de los videos con los niños. Esas preguntas tuvieron como propósito indagar si los instructores observaron cambios en los niños como consecuencia del uso de los videos en lo referente a la práctica del taekwondo, la reacción de parte de los niños a los usos de los videos, la diferencia del aprendizaje de ese deporte por parte de los niños cuando se usaron los videos y cuando no se usaron éstos, y sobre la disposición de los propios instructores a seguir utilizando videos para la enseñanza de las técnicas de ese deporte.

\section{Método}

Durante los cuatro meses que duró la investigación, a los niños se les presentaron cuatro videos sobre la explicación y ejecución correcta de: poomses -figuras- (básicos, dos videos con duración de un minuto cada uno), un video sobre la ejecución correcta de la técnica de patada (dollyo chagui -patada en redondo- y yop chagui -patada de lado-), con duración de cuatro minutos, y un último video sobre los mejores $\mathrm{KO}$ en combates con duración de cuatro minutos y 40 segundos, que fueron previamente seleccionados por los investigadores (con la orientación de un maestro en el arte del taekwondo, excampeón nacional de este deporte, y coautor de este estudio) por su contenido y pertinencia con los objetivos de la investigación. Los cuatro videos están en idioma español y fueron presentados en el mismo orden a cada grupo de niños. Los ejercicios con los niños se realizaron en las instalaciones de los respectivos colegios en horarios de sus clases formales de deporte (horas de la mañana). Durante su entrenamiento de la práctica de taekwondo, con el apoyo de video, cada grupo de niños tuvo siempre su mismo instructor, que fue el mismo que presta el servicio de instructor para los niños en su respectivo colegio.

Al finalizar los cuatro meses de las prácticas del deporte, con apoyo del video, se les aplicó a los niños una encuesta conformada por diez enunciados, en escala Likert de 1 a 5 , en donde se averiguó sobre la percepción de los niños de los beneficios del uso del video para la práctica del taekwondo. El cuestionario se elaboró a partir de la fundamentación teórica expuesta en la revisión de literatura y fue sometido a juicio de cuatro expertos. Su prueba de fiabilidad proporcionó un Alpha de Cronbach de 0,803. También, de forma simultánea, se aplicó un cuestionario similar a cada uno de los cuatro instructores de taekwondo que dirigieron el preexperimento, y a quienes, adicionalmente, se les realizó una entrevista sobre su percepción de los beneficios del uso de los videos en la práctica del taekwondo por parte de los niños. 


\section{Procesamiento de datos}

Del total de los 53 niños con que se inició el preexperimento, para este estudio sólo se analizó la información de 42 niños que asistieron a todas las prácticas durante los cuatro meses (una sesión por semana) que duró el estudio y que, además, respondieron de forma completa la encuesta de percepción del uso de los videos. El procesamiento y análisis de la información se realizó en tres niveles: en el primer nivel se determinó el grado de influencia individual que tuvieron los videos sobre cada uno de los diez factores analizados: 1. Mejorar la técnica de ejecución de las figuras, 2. Mejorar la técnica del combate, 3. Servir de guía para conocer nuevas figuras, 4. Corregir errores de ejecución en el combate, 5. Apoyar lo enseñado por parte del instructor, 6. Aprender más fácil las figuras, 7. Repasar de manera autónoma las diferentes técnicas aprendidas en clases, 8. Conocer otros ejercicios para reforzar lo aprendido, 9. Compartir lo aprendido con otros practicantes, y 10. Motivar la práctica del deporte. En el segundo nivel se seleccionó el factor que fue reconocido como el de mayor influencia y mediante pruebas chi-cuadrado de Pearson se evaluó si este factor ejerce influencia sobre los restantes nueve. Para ese propósito se aplicó la siguiente fórmula del Chi-cuadrado:

$$
\chi^{2}=\sum \frac{(O-E)^{2}}{E}
$$

\section{En esta ecuación, $\mathrm{X}^{2}$ : chi cuadrado; O: frecuencia observada; y E: frecuencia esperada}

En el tercer nivel de análisis se determinó la utilidad de los videos sobre el conjunto total de factores de aprendizaje, medida por la percepción de los niños y los profesores. De esta forma se determinó la proporción de aceptación de los videos con $\mathrm{p}<0,05 \mathrm{y}$, adicionalmente, mediante el coeficiente $\mathrm{V}$ de Cramer se estableció la contribución de cada factor a la percepción de utilidad dada por los niños. El coeficiente $\mathrm{V}$ de Cramer se calculó mediante la siguiente fórmula.

$$
v=\sqrt{\frac{x^{2}}{n(k-1)}}
$$

Donde, V: coeficiente de Cramer (indicador de asociación de variables para valores superiores 0,4 (LópezRoldán y Fachelli, 2015); $X^{2}$ : chi Cuadrado; n: total de casos; y K: es el menor valor entre filas y columnas

\section{RESULTADOS Y DISCUSIÓN}

Los resultados de este estudio se presentan en tres niveles: resultados de las encuestas a los niños sobre la experiencia del uso del video para su aprendizaje del taekwondo; resultados de la percepción de los instructores del taekwondo (participantes del estudio) con el uso del video en sus prácticas con los niños; y resultados de la entrevista a los mismos instructores sobre la reacción de los niños por el uso del video para sus prácticas de taekwondo.

\section{Resultados de la encuesta a los niños}

Los resultados descriptivos se encuentran en la tabla 1 e indican que los niños participantes del estudio identifican a la motivación hacia la práctica de este deporte como la mayor utilidad de los videos. En efecto, el $85,4 \%$ de los niños considera que los videos despiertan alta motivación y, en promedio, puntuaron este aspecto con 4,6, en una escala de 1 a 5 . En segundo lugar de importancia, con una calificación promedio de 4,4 , consideran que los videos sirven para apoyar lo enseñado por parte del instructor. En tercer lugar, con una calificación de 4,3, consideran que los videos sirven para mejorar la técnica de combate así como para conocer otros ejercicios que refuercen lo aprendido. Estos resultados concuerdan con lo dicho por Osuna (2018), Wei et al. (2015), Lu y Ying-Chieh (2014) y Di Serio (2013), quienes afirman que la incorporación de la tecnología está marcada por la motivación que el sujeto o esa tecnología generen. También confirman los planteamientos de Álvarez (2016) quien afirma que el entorno multimedia contribuye a mejorar las técnicas de ejecución de este deporte.

Con respecto a la relación entre la motivación por el uso del video y los demás beneficios de esta tecnología en la práctica del taekwondo, los resultados de la tabla 1 indican que, con significancia igual o menor de 0,05, la motivación por el uso de los videos sólo está directamente relacionada con la mejora de la técnica en la ejecución de las figuras en ese deporte y con la corrección de errores de ejecución en el combate y no con los demás beneficios identificados por el uso del video. Estos resultados contrastan con los planteamientos de Grissom et al. (2013), Bernaus et al., (2009) y Pearson y Moarnaw (2005) quienes sostienen que existe relación positiva entre la motivación generada por el alumnado frente a una estrategia pedagógica y los 
diferentes resultados de su aprendizaje, pero son coherentes con los estudios sobre los procesos de enseñanza y de aprendizaje que consideran este proceso como algo complejo que va más allá de una actividad con énfasis motivacional (Schunk y Zimmerman, 2012 y Jaclyn, 2010).

Tabla 1: Percepción de los niños con respecto a la utilidad del video en el aprendizaje del taekwondo

\begin{tabular}{|l|c|c|}
\hline Factor de aprendizaje & Promedio & Varianza \\
\hline Mejorar la técnica en la ejecución de poomses (figuras) & 3,59 & 1,249 \\
\hline Mejorar la técnica de combate. & 4,3 & 0,812 \\
\hline Servir de guía para conocer nuevos poomses (figuras). & 3,78 & 1,726 \\
\hline Corregir errores de ejecución en el combate. & 4,17 & 1,245 \\
\hline Apoyar lo enseñado por parte del instructor (profesor) & 4,44 & 1,002 \\
\hline Motivar la práctica del deporte. & 4,60 & 0,549 \\
\hline Aprender más fácil los poomses (figuras). & 3,90 & 1,290 \\
\hline Repasar de manera autónoma las técnicas aprendidas en las clases. & 3,95 & 1,248 \\
\hline Conocer otros ejercicios para reforzar lo aprendido. & 4,3 & 1,045 \\
\hline Compartir lo aprendido con otros practicantes. & 4,10 & 1,490 \\
\hline
\end{tabular}

La visión de conjunto sobre la contribución de los videos a los procesos de enseñanza y de aprendizaje del taekwondo se presenta en la tabla 2, en donde se destaca que las mayores contribuciones se dan en los factores que registran una $V$ de Cramer superior a 0,5 y $p<0,001$. Se trata de los primeros tres factores de la tabla y que tienen como característica común que los niños las pueden realizar sin la presencia de su instructor, lo cual concuerda con los planteamientos Sánchez-Otero et al. (2019) y de Grissom et al. (2013) quienes consideran que, las TIC son estrategias pedagógicas que motivan en el alumno el interés por su propio aprendizaje aún en ausencia del docente. También, los datos de la misma tabla 2 indican que los niños le dieron menos importancia al uso de los videos como estrategia para facilitar el aprendizaje autónomo y esto se debe, posiblemente, a que, por su corta edad, el aprendizaje del taekwondo les demanda esfuerzo físico y mental y la presencia del instructor les genera confianza y evidencia que el aprendizaje es un proceso complejo que involucra diversos aspectos tanto del que aprende y del que enseña como del contexto en el que se da este proceso (Martínez-Argüello et al., 2018; Schunk, y Zimmerman, 2012; Jaclyn, 2010).

Tabla 2: Relación entre la motivación percibida por los estudiantes gracias a los videos y el aprendizaje del taekwondo

\begin{tabular}{|l|c|c|}
\hline Variable evaluada & Chi Cuadrado de Pearson & Significancia \\
\hline Mejorar en la técnica en la ejecución de poomses (figuras). & 11,769 & 0,019 \\
\hline Mejorar en la técnica de combate. & 2,128 & 0,712 \\
\hline Servir de guía para conocer nuevos poomses (figuras) & 5,392 & 0,249 \\
\hline Corregir errores de ejecución en el combate & 10,637 & 0,031 \\
\hline Apoyar lo enseñado por parte del instructor (profesor) & 2,685 & 0,612 \\
\hline Aprender más fácil los poomses (figuras) & 2,041 & 0,728 \\
\hline Repasar de manera autónoma las técnicas aprendidas en clases & 6,187 & 0,186 \\
\hline Conocer otros ejercicios para reforzar lo aprendido & 2,240 & 0,692 \\
\hline Compartir lo aprendido con otros practicantes & 5,431 & 0,246 \\
\hline
\end{tabular}

\section{Resultados de la encuesta y la entrevista a los instructores}

La percepción de los instructores con respecto a la influencia de los videos sobre los diez factores de aprendizaje analizados, en este estudio, coincide con la de los estudiantes pues consideran que la motivación es el factor más reconocido, seguida por su utilidad como apoyo a lo enseñado por parte del instructor y, en tercer lugar, los videos sirven para corregir los errores de ejecución en el combate. Así mismo, perciben que los videos tienen bajo impacto en el aprendizaje autónomo, esto debido en gran parte a que, el aprendizaje no se reduce al uso de una técnica o una tecnología que puede ser motivadora, sino que, es un proceso que involucra factores personales, del contexto y del instructor (Schunk, y Zimmerman, 2012; Jaclyn, 2010; Traveset, 2007). 
Tabla 3: Contribución de los videos al mejoramiento de los factores de aprendizaje

\begin{tabular}{|l|c|c|}
\hline Factor de aprendizaje & V de Cramer & Significancia \\
\hline Conocer otros ejercicios para reforzar lo aprendido. & 0,666 & 0,000 \\
\hline Corregir errores de ejecución en el combate. & 0,527 & 0,001 \\
\hline Compartir lo aprendido con otros practicantes. & 0,525 & 0,001 \\
\hline Apoyar lo enseñado por parte del instructor (profesor) & 0,459 & 0,003 \\
\hline Mejorar la técnica de combate. & 0,458 & 0,003 \\
\hline Mejorar la técnica en la ejecución de poomses (figuras) & 0,453 & 0,004 \\
\hline Aprender más fácil los poomses (figuras). & 0,368 & 0,018 \\
\hline Repasar de manera autónoma las técnicas aprendidas en las clases. & 0,368 & 0,018 \\
\hline Guía para conocer nuevos poomses (figuras). & 0,324 & 0,038 \\
\hline Motivar la práctica del deporte. & 0,311 & 0,046 \\
\hline
\end{tabular}

El análisis integral de la influencia de los videos en el proceso de aprendizaje por parte de los instructores permitió establecer que el $75 \%$ de ellos considera que los videos son de gran utilidad, en tanto que el $25 \%$ lo considera de moderada utilidad, sin embargo, todos coinciden en que estos se constituyen en una estrategia de trabajo importante que facilita la motivación de los estudiantes y su desempeño como instructores. En síntesis, los resultados de la entrevista a los instructores respecto del uso de los videos por parte de los niños, el estudio indica que:

a) Respecto de los cambios observados en la técnica aplicada hay consenso en que se mejoró en la precisión de los movimientos y en las técnicas utilizadas por los niños para sus ejercicios y se evidenció la corrección de posturas, lo cual es coherente con la afirmación de Álvarez (2016)) quien dice que el uso del video le permite al maestro observar en sus practicantes la mejora de su técnica en la práctica de su deporte.

b) La reacción por parte de los niños ante el uso del video fue la mayor motivación para realizar más prácticas y más complejas, todo esto con una gran actitud de imitación de los protagonistas de los ejercicios en los videos. Esto es concordante con los planteamientos de Grissom et al. (2013).

c) En comparación con los estudiantes que no hicieron parte del estudio, los participantes estuvieron más motivados en la práctica de los ejercicios del deporte, realizaron mejor los movimientos y las técnicas de ese deporte, demandaron menos horas de entrenamiento para cada ejercicio y se autodefinieron metas más exigentes en sus prácticas.

\section{Efectos del instructor en los resultados del preexperimento}

De otra parte, es importante mencionar que, para determinar, si hubo efectos del instructor en los resultados del preexperimento en su respectivo grupo, se utilizó el test de Kruskal-Wallis (Chou, 1989). Los resultados de la prueba se presentan en la tabla 4 y éstos indican que, como consecuencia de haber contado con cuatro instructores en el preexperimento (uno por grupo) en general, no se presentan diferencias en los resultados de los grupos, con excepción de dos factores: mejorar la técnica en la ejecución de poomses (figuras) y conocer otros ejercicios para reforzar lo aprendido.

\section{DISCUSION FINAL}

La investigación permitió evidenciar, por un lado, que la práctica del taekwondo es una estrategia positiva en el proceso formativo de los niños en edad escolar y, de otro lado, que el uso del video como herramienta tecnológica facilita los procesos de enseñanza y de aprendizaje dado que los niños actuales son considerados nativos digitales, por lo tanto, encuentran en el uso de la tecnología una alternativa positiva y motivadora que les permite ser partícipes directos en su propio proceso formativo.

Lo más destacado en el uso de los videos, tanto por parte de los propios niños como de sus instructores, fue la alta motivación que se generó en aquellos para realizar la práctica de ese deporte. También que, al relacionar la motivación con los demás beneficios del uso de los videos, ésta sólo se relaciona de forma positiva con la mejora en la técnica en la ejecución de las figuras y la corrección de los errores de ejecución en el combate y no con los demás beneficios del uso del video que fueron puntuados como importantes por los niños y por los instructores, lo cual corrobora que los procesos de enseñanza y de aprendizaje son procesos complejo que va más allá de solo motivación. 
Tabla 4: Prueba de diferencia de medias en los factores de aprendizaje de los grupos por instructor

\begin{tabular}{|l|c|c|c|c|c|c|}
\hline \multicolumn{1}{|c|}{ Factor de aprendizaje } & \multicolumn{3}{|c|}{ Puntuación promedio } & \multirow{2}{*}{$\begin{array}{c}\text { H de Kruskal } \\
\text {-Wallis }\end{array}$} & $\begin{array}{c}\text { Significancia } \\
\text { Asintótica }\end{array}$ \\
\cline { 2 - 6 } & Grupo 1 & Grupo 2 & Grupo 3 & Grupo 4 & 0,001 \\
\hline $\begin{array}{l}\text { Mejorar la técnica en la ejecución de } \\
\text { poomses (figuras) }\end{array}$ & 2,8 & 3,5 & 3,8 & 4,5 & 15,493 & 0,348 \\
\hline Mejorar la técnica de combate. & 4,0 & 4,1 & 4,8 & 4,5 & 3,298 & 0,465 \\
\hline $\begin{array}{l}\text { Servir de guía para conocer nuevos } \\
\text { poomses (figuras). }\end{array}$ & 3,5 & 4,0 & 4,0 & 3,8 & 2,555 & 0,850 \\
\hline $\begin{array}{l}\text { Corregir errores de ejecución en el } \\
\text { combate. }\end{array}$ & 3,8 & 4,2 & 4,5 & 4,3 & 0,797 & 0,253 \\
\hline $\begin{array}{l}\text { Apoyar lo enseñado por parte del } \\
\text { instructor (profesor) }\end{array}$ & 4,3 & 4,7 & 4,9 & 4,0 & 4,077 & 0,514 \\
\hline $\begin{array}{l}\text { Motivar la práctica del deporte. } \\
\text { Aprender más fácil los poomses } \\
\text { (figuras). }\end{array}$ & 4,4 & 4,6 & 4,5 & 4,9 & 2,290 & 0,191 \\
\hline $\begin{array}{l}\text { Repasar de manera autónoma las } \\
\text { técnicas aprendidas en las clases. }\end{array}$ & 3,6 & 3,9 & 3,8 & 4,6 & 5,744 & 0,125 \\
\hline $\begin{array}{l}\text { Conocer otros ejercicios para } \\
\text { reforzar lo aprendido. }\end{array}$ & 3,8 & 4,9 & 4,3 & 3,9 & 9,933 & 0,019 \\
\hline $\begin{array}{l}\text { Compartir lo aprendido con otros } \\
\text { practicantes. }\end{array}$ & 3,5 & 4,1 & 4,6 & 4,4 & 4,353 & 0,226 \\
\hline
\end{tabular}

\section{CONCLUSIONES}

De acuerdo al trabajo presentado y a los resultados obtenidos, se pueden plantear las siguientes conclusiones principales:

1.- El uso del vídeo en la enseñanza y el aprendizaje del taekwondo por parte de los niños de edad escolar, es importante debido a que despierta en ellos una alta motivación hacia la práctica de ese deporte, refuerza las técnicas de ejecución enseñadas por el instructor y lo aprendido sobre la técnica de combate, así como otros ejercicios.

2.- La motivación que genera en los niños el uso de los vídeos en el proceso de aprendizaje del taekwondo solo se relaciona con la mejora en la técnica en la ejecución de las figuras y la corrección de los errores de ejecución en el combate, pero no con otros beneficios del uso de esa tecnología tales como conocer otros ejercicios e incentivar la práctica de ese deporte.

3.- En general, los instructores que utilizaron los videos para la enseñanza y el aprendizaje del taekwondo por parte de los niños coinciden en que los videos si incidieron en la motivación de los niños para realizar los ejercidos de ese deporte, pero que, esa motivación influyó muy poco en el aprendizaje autónomo de los niños.

\section{REFERENCIAS}

Álvarez, A., Detection of Learning Needs by Means of a Social Network and Video in a Class of French as a Foreign Language,DOI: http://dx.doi.org/10.15198/seeci.2016.39.1-16, Revista de Comunicación de La SEECI, (39), 1-16 (2016).

Bernaus, M., A. Wilson y R. C.Gardner, Teachers's motivation, classroom strategy use, student's motivation and second language achievement, Porta Linguarum, ISSN: 1697-7467, 2, 25-36 (2009).

Bridge, C. A., J. F. da Silva Santos y otros dos autores, Physical and physiological profiles of taekwondo athletes, DOI: 10.1007/s40279-014-0159-9, Sports Medicine, 44(6), 713-733 (2014).

Carbou, J., Reflexiones sobre economía y humanismo como modelo para la formación integral, Activos, ISSN: 0124-5805, 13(25), 25-30 (2016).

Casolino, E. y C. Cortis y otros cuatro autores, Physiological versus psychological evaluation in taekwondo elite athletes, International Journal of Sports Physiology and Performance, ISSN 1555-0273, 7(4), 322-331 (2012).

Chou, Y. (1989) Statistical analysis for business and economics. New York, Elsevier Publishing Company.

Córdoba, M. M., E. López Murillo, y otros dos autores, Estudiantes de la básica y media con respecto al uso de las TIC como herramientas de apoyo a su aprendizaje, Revista Trilogía, ISSN 2145-4426, 9(16), 113-125 (2017).

Da Silva, I., y E. Silva, Taekwondo adaptado como práctica pedagógica. Revista Brasileira De Futsal E Futebol, ISSN 1984-4956, 8, 299-300 (2016).

Del Moral, M., L. Villalustre y M. Niera, Oportunidades de las TIC para la innovación educativa en las escuelas rurales de Asturias, Aula Abierta, ISSN 0210-2773, 42(1), 61-67 (2014). 
Di Serio, Á., M. Ibáñez, y C. Delgado, Impact of an augmented reality system on students' motivation for a visual art course, https://doi.org/10.1016/j.compedu.2012.03.002,Computers \& Education, 68, 586-596 (2013).

García, S., ¿Desarrolla el video como objeto de aprendizaje la competencia digital en la formación del profesorado? Revista Electrónica de Lingüística Aplicada, ISSN: 1885-9089, 85 (2017).

García, S. E., Repensar la formación humana en la modernidad, https://doi.org/10.22490/issn.2539-4088, Revista De Investigaciones De La UNAD, 13(2), 149-168 (2014).

González, J., y A. González. Perfeccionismo y "alarma adaptativa" a la ansiedad en deportes de combate, Revista De Psicología Del Deporte, ISSN: 1132-239X, 26, 15-23 (2017).

Grigore, V., Implications of Temperament Characteristics in the Educational Process of Taekwondo Sportsmen, DOI: https://doi.org/10.1016/j.sbspro.2015.04.301, Procedia - Social and Behavioral Sciences, 191, 1293-1298 (2015).

Grissom, J. A., S. Loeb, y B. Master, Effective instructional time use for school leaders: Longitudinal evidence from observations of principals, https://doi.org/10.3102/0013189X13510020, Educational Research, 42, 433- 444 (2013).

Guillen, L., A., P. Herrera, e Y. A. de la Rosa, Las herramientas tecnológicas TIC como elemento alternativo para el desarrollo del componente físico. Retos: Nuevas Perspectivas De Educación Física, Deporte y Recreación, ISSN:1988204, (34), 222-229 (2018).

Huapaya, C. R., G. M. Arona, y F. A. Lizarralde, Enseñanza de la ingeniería con sistemas tutoriales inteligentes, http://dx.doi.org/10.4067/S0718-07642005000500012, Información tecnológica, 16(5), 75-78 (2005).

Jaclyn, B., Activities, Motivation, and Outcomes in a University Language Learning Environment. Pensilvania: Carnegie Mellon University. (2010).

López-Roldán, P. y Fachelli S., Análisis de tablas de contingencia. En Metodología de la Investigación Social Cuantitativa. Bellaterra, Espanya: Universitat Autònoma de Barcelona, (2015).

Lu, Su-Ju y I. Ying-Chieh, Integrating augmented reality technology to enhance children's learning in marine education, https://doi.org/10.1080/13504622.2014.911247, Environmental Education Research, 21(4), 525-541 (2014).

Martin, D. y W. Martin, Psicología experimental: Cómo hacer experimentos en psicología. México: Cengage Learning Editores, (2008).

Martínez-Argüello, L. D., F. J. Hinojo-Lucena y I. A. Díaz, Aplicación de las Tecnologías de la Información y la Comunicación (TIC) en los Procesos de Enseñanza-Aprendizaje por parte de los Profesores de Química, http://dx.doi.org/10.4067/S0718-07642018000200041, Información tecnológica, 29(2), 41-52 (2018).

Mendoza, H. y M. Placencia, Uso docente de las tecnologías de la información y comunicación como material didáctico en Medicina Humana, https://doi.org/10.1016/j.riem.2017.04.005, Investigación en Educación Médica, 135, 1-7 (2017).

Méndez-Giménez, A. El enfoque basado en autoconstrucción de materiales. El vídeo-tutorial como estrategia de enseñanza para futuros docentes. / Retos: Nuevas Perspectivas de Educación Física, Deporte y Recreación, ISSN: 19882041, 34, 311-316 (2018).

Menéndez J. I., y J. Fernández-Río, Hibridación de los modelos de Educación Deportiva y Responsabilidad Personal y Social: una experiencia a través de un programa de kickboxing educativo. / Retos: Nuevas Perspectivas De Educación Física, Deporte Y Recreación, ISSN: 1988-2041, 30, 113-121 (2016).

Monedero, J., D. Cebrian, y P. Desenne, Usabilidad y satisfacción en herramientas de anotaciones multimedia para MOOC. DOI: http://dx.doi.org/10.3916/C44-2015-06, Comunicar, 44(XXII) 55 -62 (2015).

Orozco, A., A. Pérez y F. Sierra, From the historical inheritance of motor game to the educational potential of the sport. DOI: 10.12800/ccd.v13i38.1073, Cultura, Ciencia Y Deporte, 13(38), 175-182 (2018).

Osuna J., J. Almenara y J. Castillo, La producción de objetos de aprendizaje en realidad aumentada por estudiantes universitarios Grado de aceptación de esta tecnología y motivación para su uso* Universidad De Sevilla. 23(79),12611283 (2018).

Pearson, L. y W. Moarnaw, The relationship between teacher autonomy and stress, work satisfaction, empowerment, and professionalism. Educational Research Quarterly, ISSN: ISSN-0196-5042, 29(1), 37---53 (2005).

Sánchez-Otero, M., J. García-Guiliany y otros dos autores, Estrategias Pedagógicas en Procesos de Enseñanza y Aprendizaje en la Educación Superior incluyendo Tecnologías de la Información y las Comunicaciones, http://dx.doi.org/10.4067/S0718-07642019000300277, Información tecnológica, 30(3), 277-286 (2019).

Schunk, D. H., y BV.J. Zimmerman, (Eds.), Motivation and self-regulated learning: Theory, research, and applications. Routledge, ((2012).

Tobías-Martínez, M., M. Duarte-Freitas y A. Kemczinski, Un repositorio digital de contenido fílmico como recurso didáctico, DOI http://dx.doi.org/10.3916/C44-2015-07, Comunicar, 44(XXII), 63 -71 (2015).

Wei, X., D. Weng, L, Yue y Y. Wang, Teaching based on augmented reality for a technical creative design course, https://doi.org/10.1016/j.compedu.2014.10.017, Computers \& Education, 81, 221-234 (2015).

Zambrano, J., M. R Milán y O. Estrada, Diagnóstico inicial de la virtualización educativa en la Universidad Técnica de Manabí, Ecuador. Revista Cubana De Educación Superior, ISSN 0257-4314, 36(2), 60-66 (2017). 
